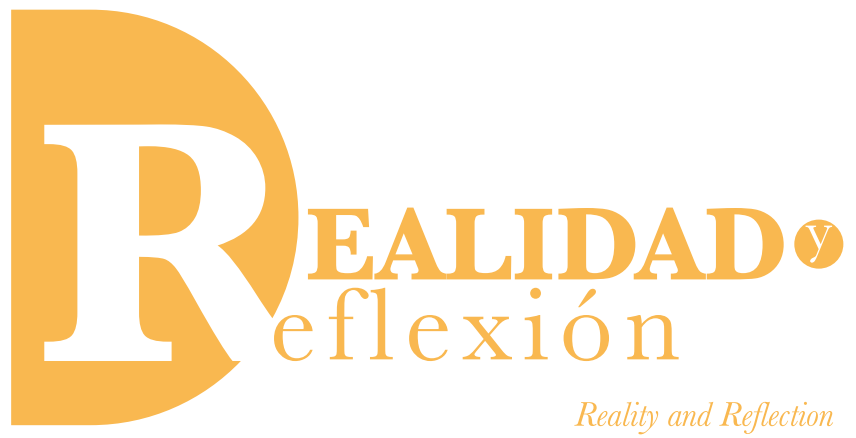

ISSN 1992-6510

Año 18, N 48, San Salvador, El Salvador, Centroamérica. Revista Semestral Julio-Diciembre 2018

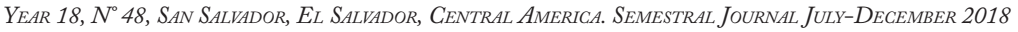

\title{
El rol de las bibliotecas nacionales en la era digital y los entornos virtuales
}

\author{
The role of national libraries in the digital age and \\ virtual environment
}

Sonia Haydée Amaya

Máster en Bibliotecas y Patrimonio Documental por la Universidad Carlos III de Madrid, España. Máster en Gestión Documental y Administración de Archivos por la Universidad Internacional de Andalucía, España. Ingeniera en Ciencias de la Computación por la Universidad Francisco Gavidia. Bibliotecóloga por la Universidad de El Salvador Directora del Sistema Bibliotecario y Docente de la Universidad Francisco Gavidia Docente de la Universidad de El Salvador samaya@ufg.edu.sv; sonhayama@gmail.com

Fecha de recepción: 10 de agosto de 2018 Fecha aprobación: 22 de noviembre de 2018 DOI:

\section{RESUMEN}

Valoración de la función de las bibliotecas nacionales frente a las tecnologías de la información y comunicaciones, se platean bondades y algunos efectos que han producido. Se identifican servicios y recursos que ofrecen algunas bibliotecas nacionales a nivel mundial en los nuevos entornos. Asimismo, se dan a conocer algunos resultados de una encuesta sobre el uso de las bibliotecas frente a la inmensa gama de posibilidades para acceder a información de interés de acuerdo a las necesidades.

Palabras clave: bibliotecas nacionales, tecnología, información y entornos virtuales.

\section{ABSTRACT}

Assessment of the function assumed by the national libraries related to the information and communication technologies, exposes benefits and some effects that have been produced. Identifies services and resources offered by some national libraries worldwide in relation to the new virtual environment. Furthermore, it presents some results of a survey about the use of libraries with overall responsibility in the immense range of possibilities to access information of interest according to the needs.

Keywords: national libraries, tecnology, information, virtual enviroment. 


\section{Introducción}

La proliferación y aplicación de las Tecnologías de la Información y Comunicación ha generado cambios en la forma de ejecutar actividades productivas, la vida de las personas y su rutina se han transformado creándose inclusive una dependencia al uso de estas, en especial Internet.

Lo anterior ha dado origen a las denominadas "generaciones", las cuales son concebidas como grupos de personas con cualidades propias y rasgos de conducta relacionados a las competencias en el uso de las TIC. En una investigación sobre la generación del milenio realizada por periódico El Diario de Hoy, se menciona que en El Salvador como en otras partes del mundo, se dan cuatro generaciones distintas: Los Baby Boomers (55-75 años), las generaciones " $\mathrm{X}$ " (35 a 45 años) y " $Y$ " (2034 años) esta última también conocida como "Millennials" y la "Z" (6-19 años) considerada como nativa digital.

Asimismo, las TIC han permitido la globalización, incremento de las economías, nuevos comportamientos sociales y culturales, innovación en la educación, surgen concepciones como "economía del conocimiento", "era de la información", "sociedad de la información y del conocimiento", "gestión de contenidos e información digital”.

Un elemento común en los términos anteriores es "información", considerada como un bien y derecho de las personas para satisfacer ciertas necesidades individuales y a la vez permite el desarrollo de cualquier nación. En la actualidad es evidente que el acceso a la información y datos ha cambiado sustancialmente desde buscarla en lugares físicos, centros específicos o comprarla mediante publicaciones ofrecidas por editores, hasta la sobreabundancia de información que está disponible en Internet.

En adelante se abordará este aspecto en relación con su accesibilidad, particularmente se tratan las unidades de información como un medio imprescindible que ha facilitado todo tipo de información según la demanda de los diferentes usuarios a los que sirven.

Se retoman bibliotecas nacionales como centros de información en torno a Internet y cómo ésta ha venido a incidir en su existencia, funcionamiento y la necesidad de reinventarse, innovar y modificar su actuar.

\section{Las bibliotecas como medios para acceder a la información}

A lo largo de la historia, desde la aparición de las primeras bibliotecas rudimentarias (conjunto de tablillas de arcilla) como las que existieron en Mesopotamia, Egipto, Siria y Babilonia que se remontan al milenio IV a.de C, han sido concebidas como una colección de libros, publicaciones periódicas u otros documentos organizados según sistemas preestablecidos y destinados a servir al público, también se definen como un edificio, local o sala donde se reúnen gran cantidad de libros.

Desde esos tiempos hasta nuestros días, las bibliotecas han venido desarrollándose y con el surgimiento de las TIC aproximadamente hace más de 38 años; se introduce tecnología, automatización de sus procesos y mejora en algunos servicios. 
Sin embargo, esto se hace más notable con el surgimiento de Internet, que ha permitido hacerlas más visibles ante el mundo, crear servicios no presenciales y facilitar el acceso a la información desde cualquier lugar y en el momento que se necesite, dando origen a los catálogos en línea, bibliotecas virtuales, digitales, repositorios institucionales, repositorios de datos investigación, cosechadores de repositorios hasta la creación de las denominadas Ciberotecas y sumado a todos los recursos libres que encontramos a través de los navegadores llegamos a lo que se denomina sobreabundancia de información, también conocido como "infoxicación", abriendo las posibilidades para los demandantes de información con diferentes perfiles según la generación a la que pertenecen. Ante lo anterior surge la interrogante ¿desaparecerán las bibliotecas?, ¿deben reinventarse?, ¿deben cambiar su rol?, y en específico en este caso de estudio las bibliotecas nacionales.

Según algunas investigaciones y noticias; en el 2011 en el artículo Un país sin bibliotecas del diario digital público se anunciaba que en "todas las ciudades en Estados Unidos; grandes, medianos y pequeños núcleos urbanos, se estaban viendo obligadas a cerrar sus bibliotecas públicas o, al menos, limitar su horario hasta la mínima expresión". En el mismo documento se planteaba como justificante el recorte de presupuestos y la tendencia a la baja en el uso de las bibliotecas debido a la rápida expansión de Internet, ya que los ciudadanos comenzaron a buscar respuestas en la forma más rápida y conveniente.

De manera similar en el 2015, Asociaciones de Bibliotecarios de Castilla-La Mancha se manifestaban ante el desmantelamiento paulatino de la Red de Bibliotecas Públicas de Castilla-La Mancha. El cierre de bibliotecas municipales, en este caso, los hechos no se justifican propiamente por el uso de Internet para la obtención de información y al conocimiento, simplemente las bibliotecas no les generan una rentabilidad económica sino un gasto, pero no evalúan la utilidad desde el punto de vista cultural.

Aunque no todos los casos relacionados al cierre de las bibliotecas estén vinculados al uso de Internet como principal medio de obtención de información, se puede mencionar que es una realidad la migración de muchos usuarios de las bibliotecas, que han pasado de usar fuentes de información soporte papel al uso de recursos disponibles en la web, trayendo como consecuencia una disminución en el uso de las bibliotecas.

Por citar un ejemplo, los estudiantes universitarios están haciendo uso de sitios web gratuitos donde encuentran la información según sus necesidades, videos sobre cualquier temática de interés, aplicaciones móviles que les son de utilidad en la vida diaria como en su formación académica; sin embargo, en mucho de los casos en especial énfasis en la información no se hace un uso efectivo de la misma o los usuarios carecen de habilidades informativas, lo que hace más difícil la calidad de su trabajo investigativo. Al realizar una encuesta o sondeo de opinión a una muestra de estos estudiantes para tratar de obtener una visión sobre el futuro de las bibliotecas en cuanto a su existencia y uso en relación con Internet se obtuvieron los siguientes resultados: 
$\mathrm{E} 170 \%$ de los encuestados que respondieron son hombres y mayores de 26 años, al consultarles qué tipo de biblioteca ha visitado un $90 \%$ respondió que la biblioteca de su universidad, a la interrogante cómo deberían ser las bibliotecas en la actualidad expresaron: con mayores espacios, más innovadores y con tecnología, 100 $\%$ virtuales, deben ofrecer la información con mayor rapidez y contar con personal capacitado que les apoye. Al preguntarles si las bibliotecas desaparecerán, el $90 \%$ dijo que no, como argumentos dieron los siguientes motivos: en las bibliotecas hay información valiosa que los medios electrónicos no tienen; hay información especializada; no desaparecerán pero deberán actualizarse, porque la lectura en libros impresos es mucho mejor que la lectura electrónica.

En relación con los recursos bibliográficos que utilizan con más frecuencia para el desarrollo de sus trabajos de investigación o practicar la lectura, el 60\% usa Internet incluyendo libros electrónicos y acceden a estos recursos desde una PC o laptop.

$\mathrm{Al}$ analizar los datos reflejados en este sondeo de opinión, la edad de los estudiantes oscila entre la generación " $\mathrm{X}$ ”, para quienes su niñez fue análoga y en su madurez tuvieron que adaptarse a la vida digital y la Millennials cuyas características es que se convirtieron en mayores de edad con la entrada del nuevo milenio y apenas recuerdan cómo era la vida sin Internet; ellos expresan, en un 90\%, que las bibliotecas no desaparecerán pero se debe innovar y contar con más tecnología, ser $100 \%$ virtuales y ofrecer la información con mayor rapidez; sin embargo, es un poco contradictorio decir que la lectura en físico es mejor que en formato electrónico.

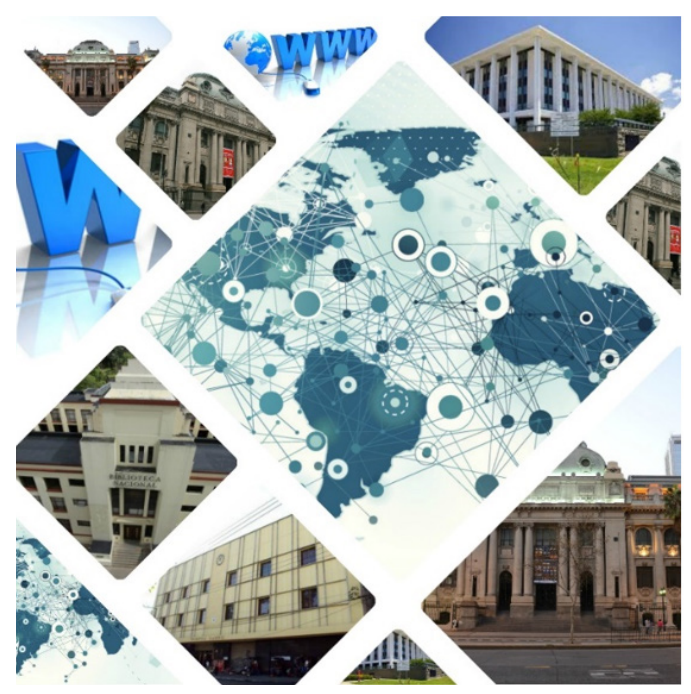

Fig. n. ${ }^{\circ}$ 1. Las bibliotecas nacionales y su quehacer en la era digital. Fuente: elaboración propia y fotografías de bibliotecas que se encuentran disponibles en web.

Todas las bibliotecas nacionales justifican en su existir un común denominador que es conservar y divulgar el patrimonio bibliográfico de un país al servicio de sociedad. Retomando la misión de algunas bibliotecas nacionales se evidencia lo escrito: "Contribuir al fortalecimiento de nuestra identidad cultural, recopilando, organizando, conservando y difundiendo el patrimonio bibliográfico salvadoreño, para que sirva como medio informativo e investigativo que impulse esfuerzos nacionales dirigidos a elevar el nivel social, económico y educativo de la población" Biblioteca Nacional de El Salvador.

"Preservar y transmitir el patrimonio bibliográfico y documental español. Su voluntad es revertir en la sociedad el inmenso legado 
cultural que atesora, potenciando al máximo el acceso, uso y reutilización de la información que conserva y genera, en beneficio de la sociedad", Biblioteca Nacional de España.

"Garantiza la recuperación, preservación y acceso a la memoria colectiva del país, representada por el patrimonio bibliográfico y hemerográfico en cualquier soporte físico. Es también la entidad nacional a cargo de la planeación y diseño de políticas relacionadas con la lectura y las bibliotecas públicas, así como de su promoción y fomento" Biblioteca Nacional de Colombia.

Se constata que es importante y necesaria la existencia de estos espacios como fuentes de información para estudiantes, investigadores o cualquier ciudadano que necesita conocer sobre la cultura de un país, pero también es una necesidad que en la denominada era digital, en la cual subsistimos, con el predominio de producción de recursos de información electrónicos, deben ser transformadas e innovadas constantemente para mantenerse visibles en la red; en este sentido, requieren aplicar tecnologías para abrir el conocimiento al mundo y satisfacer necesidades de las actuales y nuevas generaciones que buscan información contenida en las colecciones que con tanto empeño custodian; así como demás aspectos relacionados al acceso a la información.

Con el objetivo de conocer la percepción de los usuarios que hacen uso de la Biblioteca Nacional de El Salvador también se suministró una encuesta con los mismos aspectos que se consideraron para los estudiantes universitarios sobre el uso de las bibliotecas en relación con Internet y se obtuvieron los siguientes resultados:
E1 44.1\% de los usuarios encuestados fueron hombres, más del $77 \%$ son mayores de 26 años; al consultarles qué tipo de biblioteca ha visitado un 88.9 \% respondieron que visitan la biblioteca nacional. A la interrogante de cómo deberían ser las bibliotecas en la actualidad expresaron: tener todos los libros impresos, pero también digitales, que tengan información real de El Salvador un poco más actualizada con tecnología que llamen más la atención del público, que esta tecnología sea amigable para los usuarios, que tengan buena atención y sean accesibles para los usuarios; es decir, que faciliten la consulta de los acervos; que puedan abrir un poco más el recurso tecnológico y que la información esté bien clasificada, con datos completos sobre los libros disponibles.

Al preguntarles si las bibliotecas desaparecerán, un $67.7 \%$ dijo que "NO", argumentando su opinión con los siguientes motivos: nunca hay suficientes libros en Internet, pues las bibliotecas nos dan una información más puntual y nos ayuda más a la lectura; siempre es necesaria la lectura en físico, siempre nos ayudan, porque hay documentos antiguos como periódicos que en muchos casos no están disponibles en Internet.

En relación con el recurso bibliográfico que utiliza con más frecuencia para el desarrollo de sus trabajos de investigación o practicar la lectura, el $66.7 \%$ usa Internet incluyendo libros electrónicos y lo hacen accediendo desde una PC o laptop. 


\section{¿Considera que las bibliotecas desaparecerán?}

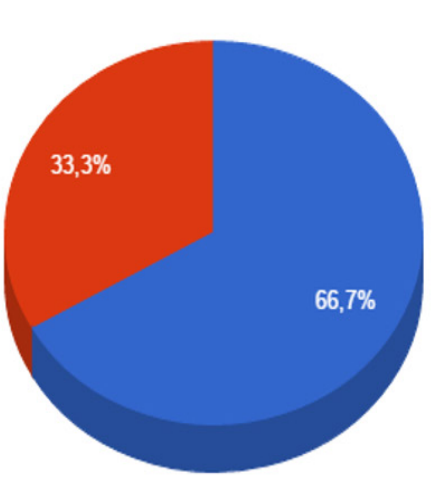

\section{Para el desarrollo de sus trabajos de investigación o practicar la lectura ¿qué recurso utiliza con más frecuencia?}

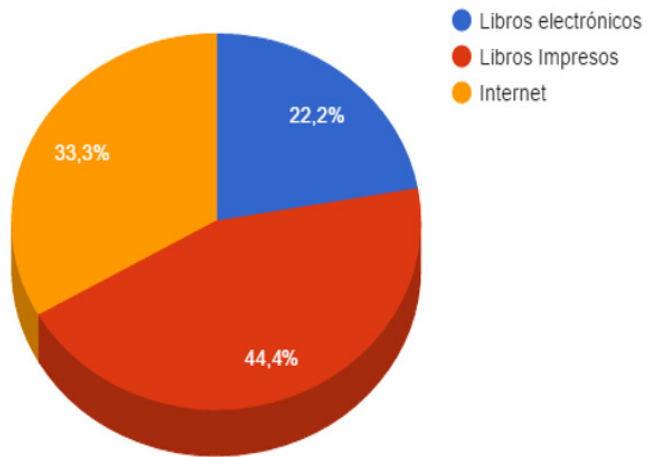

También se consultó a los usuarios de la Biblioteca Nacional cuál es el medio por el que ha visto o escuchado publicidad sobre los servicios que ofrece y las actividades culturales que realiza la Biblioteca Nacional de El Salvador y un $67.7 \%$ dijo que por la televisión, un 22.2 $\%$ por redes sociales y el otro $22.2 \%$ por la web.

Al analizar los datos reflejados por los encuestados, quienes son estudiantes e investigadores que visitan y hacen uso de la Biblioteca Nacional de El Salvador, pertenecientes a las generaciones "X" y Millennials; expresaron que las bibliotecas no desaparecerán ya que consideran que no hay suficientes libros en Internet y que siempre es necesarioleer en físico pero que deben actualizarse con tecnología, ser bibliotecas híbridas que combinen lo físico con lo digital, y un aspecto que recalcaron fue la importancia de recibir una muy buena atención y un buen servicio de parte de los bibliotecarios, enfatizando la importancia del factor humano.

Tomando en consideración las opiniones anteriores, muchas bibliotecas nacionales han mostrado un avance en mejorar sus servicios, han puesto a disposición, a través de la web, colecciones a texto completo, lo que ha dado la pauta para la crear repositorios, bibliotecas digitales o virtuales. Algunas iniciativas son: The European Library, Open Library, Europeana Collections, Biblioteca Virtual Miguel de Cervantes, Biblioteca Digital Hispánica, Ciberoteca, entre otras. Estos proyectos sumados a la edición de libros electrónicos, las plataformas de préstamos digitales y las alianzas estratégicas para aumentar los recursos de información en la web han permitido que ofrezcan muchas más fuentes de información digitales que impresas.

Sin embargo, para que puedan mantenerse firmes y no desaparecer en el mundo del Internet, las bibliotecas deben seguir reinventándose $\mathrm{o}$ transformándose de una manera constante, es necesario seguir innovando; crear más servicios en línea con la aplicación de herramientas de la Web 3.0; diseñar aplicaciones móviles que posibiliten el acceso a estas bibliotecas en el momento que los usuarios lo requieran de una forma rápida y sencilla; aplicación de la gamificación; espacios 
físicos innovadores que permitan el trabajo en equipo, crear y socializar entre los usuarios (Learning Commons, Makerspace); elaboración de planes de marketing incluyendo todos los medios de comunicación sociales actuales que permitan promover el quehacer y la gama de servicios que ofrecen; convertirse en bibliotecas híbridas o completamente digitales, siempre y cuando exista una legislación que respalde los derechos de autor de los creadores de los documentos; también es ineludible fortalecer el trabajo colaborativo con organismos o instituciones que apoyen el quehacer y proyectos de desarrollo de las bibliotecas.

Asimismo, se necesita contar con bibliotecarios que se adapten a los constantes cambios, se desempeñen como docentes, informáticos $\mathrm{y}$ ejecuten programas enfocados al desarrollo de habilidades informativas hacia los usuarios y que les permitan a estos seleccionar la información adecuada ante la sobreabundancia de datos en la red. Son necesarios bibliotecarios investigadores que apoyen a este sector en el uso de herramientas diseñadas especialmente para ellos. Se necesita de bibliotecarios con esa visión de transformación y actitud emprendedora que facilita el estar siempre activo y visible al mundo, no menos importante es contar con personas con actitud de servicio que permitan crear bibliotecas con funciones de responsabilidad social, personas interesadas en la lectura y conocedoras de diversos temas y áreas de conocimiento para crear espacios como las bibliotecas humanas (el consultar personas en lugar de libros).

Otro factor de importancia es llevar a cabo, en una forma constante, benchmarking que permita analizar y aplicar buenas prácticas que existen en otras bibliotecas nacionales.
En el artículo consultado la Red de Bibliotecas en Finlandia, país que se caracteriza por la calidad de su educación, se indican algunas de las funciones que desarrolla esta red, las que son muy similares a las expuestas anteriormente: "Asegurar la disponibilidad de unos servicios bibliotecarios e informáticos diversificados y de alta calidad en todo el país y a nivel mundial, fomentar la producción de servicios y contenidos digitales, consolidar el papel de las bibliotecas para ampliar los conocimientos básicos de la sociedad informatizada, fomentar la adquisición de competencias y destrezas informacionales en colegios y bibliotecas públicas, desarrollar gradualmente en el nivel nacional un portal informático común a todo tipo de bibliotecas”.

En este mismo sentido, es necesario realizar vigilancia tecnológica y del entorno que posibilite analizar las diferentes situaciones que afectan positiva o negativamente a las bibliotecas.

En el artículo El ascenso y caída de los eBooks, publicado en el 2018 en un periódico nacional, se expone que para el año 2015 se esperaba que la venta de libros electrónicos superara al físico; sin embargo, la venta de libros electrónicos había dejado de crecer y en 2016 decreció en $18 \%$ con respecto al anterior, también se menciona que los libros infantiles siguen siendo más populares y académicamente más útiles consumirlos en impreso, factor a considerar en las bibliotecas nacionales, puesto que varias de ellas llevan como parte de su misión la promoción y fomento a la lectura.

Sin embargo, para cumplir con todas estas funciones de innovación y transformación es necesario, además de contar con el pensamiento digital y el ímpetu de los responsables que las lideran, el poseer 
los recursos necesarios, el apoyo gubernamental y demás organizaciones internacionales.

A manera de conclusión, se puede mencionar que no hay tecnología si no hay personas, la mezcla entre estas variables es un aspecto importante en nuestro mundo bibliotecario, las fuentes de información irán cambiando de acuerdo a las nuevas tecnologías, el desarrollo de cada nación y las diferentes generaciones, las bibliotecas no desaparecerán se transformarán, incluyendo proyectos como las bibliotecas humanas y demás innovaciones.

\section{Referencias bibliográficas}

Alcaraz, G. B. (2012). Ser estudiante en la sociedad de la información y el conocimiento: la escuela y sus actores ante el cambio cultural. (Spanish). Sinéctica, (38), 1-19.

Arévalo, J. (2016). Papel de los bibliotecarios en la gestión de datos de investigación. Universo Abierto. Consultado el 5 de noviembre de 2018, Disponible en https://universoabierto. org/2016/08/30/papel-de-los-bibliotecariosen-la-gestion-de-datos-de-investigacion/

Arnaz, R.(2011).Unpaíssinbibliotecas. Consultado el 11 de julio 2016. Disponible en: http://www. publico.es/culturas/pais-bibliotecas.html

Biblioteca Nacional de Colombia. (2016). Misión y visión. Disponible en: http://www. bibliotecanacional.gov.co/content/mision_vision

Biblioteca Nacional de España. (2016). Información institucional. Disponible en http:// www.bne.es/es/Inicio/
Calero,Á.(2018).Elascenso y caída de los eBooks. La Prensa Gráfica, pág. 1. Disponible en https:// www.laprensagrafica.com/techlife/El-ascensoy-caida-de-los-eBooks-20181118-0001.html

El Diario de Hoy. (2016). Mente Millennial: una investigación de la generación del milenio. 22 p.

European Commission. (2018). Turning fair into reality: final Report and Action Plan from the European Commission Expert Group on FAIR Data. Disponible en: https://ec.europa.eu/info/ sites/info/files/turning_fair_into_reality_1.pdf

Gamero, A. (2016). Las bibliotecas humanas donde se consultan personas en lugar de libros. Disponible en http://lapiedradesisifo. com/2016/04/05/1as-bibliotecas-humanasdonde-se-consultan-personas-en-lugar-delibros/

Gilardoni, C. (2014). La Red de Bibliotecas en Finlandia. Infotecarios. Disponible en: http:// www.infotecarios.com/la_red_de_bibliotecas_ en_finlandia/

Hernández-Pérez, T, \& García-Moreno, M. A. (2013). Datos abiertos y repositorios de datos: nuevo reto para los bibliotecarios. El profesional de la información, 2013, mayo-junio, v. 22, n. 3. Disponible en: http://eprints.rclis.org/19524/1/ postprint_Hernandez_Garcia.pdf

López Bermúdez,J. G. (2016). Las 15 bibliotecas digitales que debemos conocer. Infotecarios. Disponible en: http://www.infotecarios.com/ las-15-bibliotecas-digitales-que-debemosconocer/ 
López, L. N. (2012). De la arcilla al e-book historia del libro y las bibliotecas. Disponible en: http:// eprints.rclis.org/17420/1/NOVELLE\%20 LÓPEZ,\%20LAURA\%20-\%20De\%201a\%20 arcilla\%20a1\%20E-book.pdf

Los lectores usan más el libro tradicional frente al libro electrónico. Consultado el 15 de julio de 2016. Disponible en http://leer.es/observatorio/ destacados/-/asset_publisher/VSJd7SIb1U5x/ content/id/564212

Martínez de Sousa, J. (1993). Diccionario de bibliología y ciencias afines. Madrid: Fundación German Sánchez Ruipérez.
Pacho Ferreras, L. (2010). Las TIC, realidad y perspectivas en la economía española. Partida Doble, 21(217), 24-33.

Biblioresistenciaclm. (2016). Profesionales bibliotecari@s de castilla-la mancha en defensa de las bibliotecas públicas de pequeñas y grandes poblaciones, su labor social y cultural. Biblioresistenciaclm.blogspot.com. Disponible en: http://biblioresistenciaclm.blogspot.com/p/leeaqui-el-manifiesto.html 